\title{
Lorenz Benzeri Doğrusal Olmayan Üç Boyutlu Yeni Bir Diferansiyel Denklem Sisteminin Kararlılık Analizi
}

\author{
Yener ALTUN*, Abdullah YİĞİT \\ Van Yüzüncü Yll Üniversitesi, Fen Fakültesi, Matematik Bölümü, Van \\ (ORCID: 0000-0003-1073-5513) (ORCID: 0000-0002-0099-3095)
}

\begin{abstract}
$\ddot{O} z$
Kaotik sistemler, başlangıç koşullarına duyarlı ve ölçülemeyecek karmaşıklıktaki dinamik sistemler olarak ifade edilebilir. Başlangıç koşullarına olan duyarlılığının yanında kaotik sistemler, geniş bantlı ve periyodik olmayan bir özelliğe sahiptir. Bu özelliklerinden dolayı söz konusu bu sistemler, özellikle mühendislik alanlarında olmak üzere farklı bilim dallarında geniş uygulama alanına sahiptir. Bu makalede Lorenz benzeri doğrusal olmayan üç boyutlu yeni bir diferansiyel denklem sisteminin kararlılığı araştırılmıştır. Çalışmada öncelikle dikkate alınan sistemin denge noktaları belirlenmiş ve kararlılık kriterleri Hurwitz koşulları kullanılarak incelenmiş̧ir. Daha sonra, bu sistemin üstel kararlılı̆ı için gerek ve yeter koşullar tartışılmıştır. Sonuç olarak, elde edilen sonuçlar ilgili literatürde bulunan sonuçları içerir ve geliştirir.
\end{abstract}

Anahtar kelimeler: Lineer (doğrusal) olmayan sistem, Hurwitz teoremi, Kararlılık, Lyapunov fonksiyonu.

\section{Stability Analysis of a New Differential Equation System of Lorenz-like Nonlinear Three-Dimensional}

\begin{abstract}
Chaotic systems can be described as immeasurably complex dynamic systems which are sensitive to initial conditions. In addition to its sensitivity to initial conditions, chaotic systems have a broadband and non-periodicity. Because of these properties, these systems considered have a wide application area in different sciences, especially in engineering subjects. In this paper, the stability of a new differential equation system of Lorenz-like nonlinear three-dimensional, was investigated. In the study, firstly, the equilibrium points of the system which was taken into consideration were determined and the stability criteria were examined by using Hurwitz conditions. Then, the necessary and sufficient conditions for exponential stability of this system have been discussed. Consequently, the obtained results include and improve the results found in the related literature.
\end{abstract}

Keywords: Nonlinear system, Hurwitz theorem, Stability, Lyapunov function.

\section{Giriş}

Dinamik sistemler lineer veya lineer olmayan yöntemler kullanılarak incelenirler. Düzgün davranışa sahip dinamik sistemlerde çok küçük parametre değişikliği yapılarak sistem kaosa sürüklenmektedir. Kaos kavramının bilim tarihine girişi 18. yüzyıla dayanmaktadır. Başlangıç koşullarına hassas bağlılık gösteren kaos teorisi günümüzde ekolojik sistemler, fizik, kimya, mühendislik, tıp, ekonomi, iletişim güvenliği vb. çeşitli alanlara uygulanmıştır [1]. 1963’te Lorenz iki kuadratik terim ile karmaşık (kompleks) dinamik davranışlar sergileyen üçüncü mertebeden otonom ilk kaotik sistemi buldu [9].

Kaotik sistem denklemlerle tanımlanıyorsa deterministtik kaos olarak ifade edilir. Kaotik sistemlerin matematiksel modelleri lineer olmayan bir özelliğe sahiptir. Sürekli zamanlı diferansiyel denklemlerde tanımlandığı gibi ayrık zamanlı fark denklemleri ile de tanımlanabilmektedir. Söz konusu denklem sistemlerinin matematiksel modellerinin olması determinizm kavramını ortaya koyarken uzun zaman içindeki davranışın başlangıç koşullarına hassaslık göstermesi; kaotik sistemleri lineer olmayan

"Sorumlu yazar: veneraltun@yyu.edu.tr

Geliş Tarihi: 20.01.2019, Kabul Tarihi: 01.07.2019 
sistemler altında ele alınan birçok modelden farklı kılmaktadır. Basit olmasına karsın kaotik sistem modelleri davranışları oldukça karmaşıktır [13].

Kaos araştırması için Lorenz sistemi ilk kaotik model olarak bir modül haline geldi. Bu doğrultuda Chen sistemi [18], Rössler sistemi [16] ve Lü sistemi [12] gibi birçok kaotik sistem bulunmuştur. Bu kaotik sistemler pek çok araştırmacı tarafindan sıklıkla incelendi [1, 3, 5-12, 15-22].

Aşağıda ilgili literatürde çalışılmış bazı kaotik sistemler ve belirli notalardaki kaotiklik durumları belirtilmiştir:

$$
a>0, b>0, c>0 \text { için, } \begin{aligned}
& \\
\dot{x} & =a(y-x), \\
\dot{y} & =c x-x z-y, \\
\dot{z} & =x y-b z,
\end{aligned}
$$

şeklindeki Lorenz sistemi, lineer olmayan bir diferansiyel denklem sistemidir ve $(a, b, c)=(10,8 / 3,28)$ değerleri için kaotiktir [6].

$$
a, b, c \in \mathfrak{R}^{3} \text { için, } \quad \begin{aligned}
& \\
& \dot{x}=a(y-x), \\
& \dot{y}=(c-a) x-x z+c y, \\
& \dot{z}=x y-b z,
\end{aligned}
$$

şeklindeki Chen systemi, lineer olmayan bir diferansiyel denklem sistemidir ve $(a, b, c)=(35,3,28)$ değerleri için kaotiktir [8].

$$
\begin{aligned}
& a>0, b>0, c>0 \text { için, } \\
& \dot{x}=a(y-x), \\
& \dot{y}=c y-x z \text {, } \\
& \dot{z}=x y-b z,
\end{aligned}
$$

şeklindeki Lü sistemi, lineer olmayan bir diferansiyel denklem sistemidir ve $(a, b, c)=(36,3,20)$ değerleri için kaotiktir [20].

$$
\sigma>0, r>0, \beta>0 \text { için, } \begin{aligned}
& \\
\dot{x} & =\sigma(y-x), \\
\dot{y} & =r x-x z, \\
\dot{z} & =x y-\beta z,
\end{aligned}
$$

şeklindeki Pan sistemi, lineer olmayan bir diferansiyel denklem sistemidir ve $(\sigma, r, \beta)=(10,16,8 / 3)$ değerleri için kaotiktir [1].

Biz bu çalışmada $a>0, b, c \in(-\infty,+\infty)$ için,

$$
\begin{aligned}
& \dot{x}=y z-b x, \\
& \dot{y}=a(z-y), \\
& \dot{z}=c y-x y-z
\end{aligned}
$$

diferansiyel denklem sistemini inceledik. Bu sistem Lorenz benzeri üç boyutlu lineer olmayan bir diferansiyel denklem sistemidir. Bu sistemin katsayı parametrelerinin tamamı pozitif olmadığı için Lorenz denklem sistemine göre daha karmaşık bir yapıya sahiptir. Ancak söz konusu sistemin kaotik olma durumları katsayı parametrelerinin pozitif değerleri için sağlanmıştır. Burada $(a, b, c)=(25,8 / 3,30)$ değerleri için (1) denklem sistemi kaotiktir (Şekil 1.). 


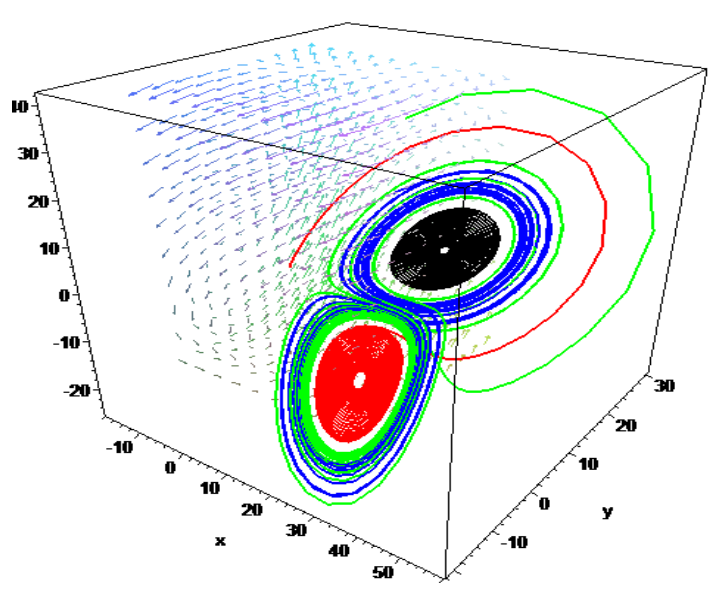

(a)

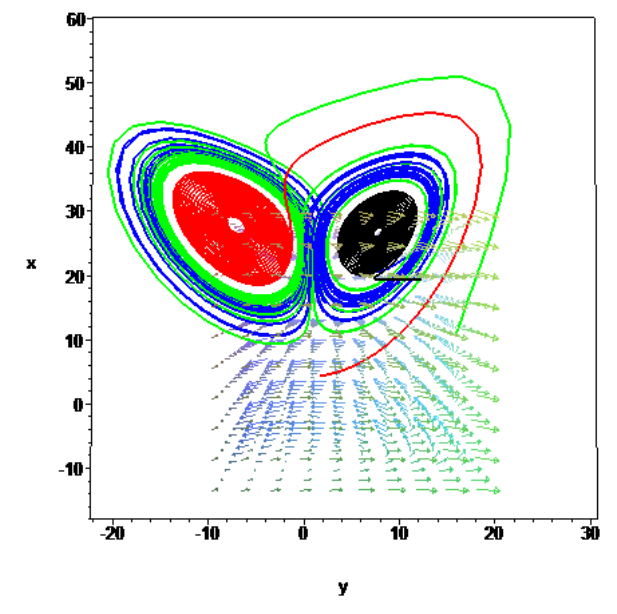

(b)

Şekil 1. (1) sisteminin üç boyutlu xyz-izdüşümü (a) ve xy-izdüşümü (b).

Bu makalede ilk olarak, (1) denklem sisteminin ayrık (isolated) denge noktalarını bulduk. Daha sonra Lyapunov fonksiyonu yardımıyla dikkate aldığımız (1) sisteminin global üstel kararlılığı için gerek ve yeter şartlar oluşturduk. Son olarak, Hurwitz teoremini kullanarak (1) sisteminin asimptotik kararlılık ve kararsızlık kriterlerini inceledik.

Ana sonucumuzu belirtmeden önce aşağıdaki temel tanımlar ve teoremden yararlanacağız.

\section{Temel Teorem ve Tanımlar}

Tanım 1. $D, \mathfrak{R}^{n}$ de orijini içeren bir bölge olsun. Ayrıca $W:[0, \infty) \times D \rightarrow[0, \infty)$ fonksiyonu verilsin. Eğer $t \geq 0$ için, $W(t, 0)=0$ ve $x \neq 0$ için, $W(t, x)>0$ ise $W$ pozitif tanımlıdır denir. Öte yandan pozitif tanımlı $W$ fonksiyonu, birinci mertebeden sürekli kısmi türevlere sahip ise $W$ ye bir Lyapunov fonksiyonu denir [2].

\section{Tanım 2.}

$\left\{\begin{array}{l}\frac{d x}{d t}=P(x, y) \\ \frac{d y}{d t}=Q(x, y)\end{array}\right.$

otonom sistemi verilsin. Herhangi bir $\left(x_{0}, y_{0}\right)$ noktasinda $P\left(x_{0}, y_{0}\right)=0$ ve $Q\left(x_{0}, y_{0}\right)=0$ ise, $\left(x_{0}, y_{0}\right)$ noktasina (2) sisteminin kritik (denge) noktası denir [14].

\section{Tanım 3. Eğer}

$$
\left(x-x_{0}\right)^{2}+\left(y-y_{0}\right)^{2}=r^{2}
$$

olacak şekilde çember içinde (2) sisteminin $\left(x_{0}, y_{0}\right)$ noktasından başka bir kritik noktası yok ise, $\left(x_{0}, y_{0}\right)$ noktasına (2) sisteminin ayrik (isolated) denge noktası denir [14].

Tanım 4. $f: \Re^{n} \rightarrow \Re^{n}$ sürekli bir fonksiyon ve $\frac{d x}{d t}=f(x), x \in \mathfrak{R}^{n}$

olsun.

a. Eğer $f\left(x^{*}\right)=0$ ise, $x^{*}$ noktası (3) için sürekli bir denge noktasıdır; 
b. $B\left(x^{*}, r\right)$, merkezi $x^{*}$ ve yarıçapı $r$ olan bir açık yuvar olmak üzere; her $t>0$, için $B\left(x^{*}, \varepsilon\right)$ de bulunan ve $x(0)=x_{0}$ ile verilen dinamik sistemin bir çözümü $x(t)$ olsun. Eğer her $\varepsilon>0$ için, bir $\delta>0$ sayısı var ise bu takdirde her $x_{0} \in B\left(x^{*}, \delta\right)$ için (3) sisteminin $x^{*}$ denge noktası kararlıdır;

c. Ĕger $x(0) \in B\left(x^{*}, \delta\right)$ ile verilen her $x(t)$ çözümü için,

$$
\lim x(t)=x^{*}
$$

olacak şekilde yeterince küçük bir $\delta>0$ sayısı var ise (3) sisteminin $x^{*}$ denge noktası asimptotik kararlıdır;

d. Eğer $x(0) \in B\left(x^{*}, \delta\right)$ ile verilen her $x(t)$ çözümü için;

$\left\|x(t)-x^{*}\right\| \leq \mu\left\|x(0)-x^{*}\right\| e^{-\eta t}, \quad t \geq 0$

olacak şekilde $\mu>0, \eta>0$ sabitleri ve $\delta>0$ sayısı var ise, (3) sisteminin $x^{*}$ denge noktası üstel kararlıdır.

Ayrıca (3) sisteminin bütün $x(t)$ çözümleri için (4) eşitsizliği sağlanırsa, $x^{*}$ denge noktasına global üstel kararlıdır denir [4].

Teorem 2.1 (Hurwitz teoremi) Reel katsayıl1

$$
P(\lambda)=\lambda^{n}+a_{1} \lambda^{n-1}+a_{2} \lambda^{n-2}+\ldots+a_{n-1} \lambda+a_{n}
$$

karakteristik polinomunun tüm köklerinin negatif reel kısımlı olabilmesi için gerek ve yeter şart

$$
H_{n}=\left[\begin{array}{ccccccccc}
a_{1} & 1 & 0 & 0 & 0 & 0 & 0 & \ldots & 0 \\
a_{3} & a_{2} & a_{1} & 1 & 0 & 0 & 0 & \ldots & 0 \\
a_{5} & a_{4} & a_{3} & a_{2} & a_{1} & 1 & 0 & \ldots & 0 \\
\vdots & \vdots & \vdots & \vdots & \vdots & \vdots & \vdots & \vdots & \vdots \\
0 & 0 & 0 & 0 & 0 & 0 & 0 & 0 & a_{n}
\end{array}\right]
$$

Hurwitz matrisinin tüm köşegen minörlerinin pozitif olmasıdır.

Not edilmelidir ki; $H_{n}$ Hurwitz matrisinin esas köşegeni, $P(\lambda)$ polinomunun $a_{1}$ den $a_{n}$ ye kadar katsayılarını içermektedir. Aşă̆ıda

$$
D_{1}=\left|a_{1}\right|, D_{2}=\left|\begin{array}{cc}
a_{1} & 1 \\
a_{3} & a_{2}
\end{array}\right|, \ldots, D_{n}=\operatorname{det}\left(H_{n}\right)
$$

ile Hurwitz matrisinin esas köşegen minörleri tanımlanır. Eğer $n=3$ ise, Hurwitz koşulları

$$
a_{1}>0, a_{2}>0, a_{3}>0 \text { ve } a_{1} a_{2}-a_{3}>0
$$

durumuna indirgenir [15].

\section{Ana Sonuçlar}

Önerme 3.1. (1) sistemini gözönüne alalım. Eğer $b \neq 0$ ve $b(c-1)>0$ ise, (1) denklem sistemi $O(0,0,0), \quad A_{1}(c-1, \sqrt{b(c-1)}, \sqrt{b(c-1)})$ ve $A_{2}(c-1,-\sqrt{b(c-1)},-\sqrt{b(c-1)})$ gibi üç ayrık denge noktasına sahip olur. Eğer $b \neq 0$ ve $b(c-1) \leq 0$ ise, (1) denklem sistemi yalnız bir $O(0,0,0)$ ayrık denge noktasina sahip olur.

İspat: (1) sisteminin denge noktaları aşağıdaki

$$
\left\{\begin{array}{c}
y z-b x=0 \\
a(z-y)=0 \\
c y-x y-z=0
\end{array}\right.
$$

denklem sisteminin çözümünden elde edilebilir. $b \neq 0$ için (5) sistemi 


$$
\left\{\begin{array}{c}
x=y z / b \\
z=y \\
c y-x y-z=0
\end{array}\right.
$$

olarak yazılabilir. Burada gerekli cebirsel işlemler yapılarak $x$ ve $z$ yok edilirse,

$$
y\left(c-\frac{y^{2}}{b}-1\right)=0
$$

olur. Bu denklem çözüldüğünde $y$ değerleri için; $b \neq 0$ ve $b(c-1) \leq 0$ iken $y=0$ olur. Öte yandan $b \neq 0$ ve $b(c-1)>0$ iken $y=0$ ve $y= \pm \sqrt{b(c-1)}$ olur. Bu yüzden (1) sistemi, $b \neq 0$ ve $b(c-1) \leq 0$ iken $O(0,0,0)$ şeklinde tek bir denge noktasına sahip olur. Ancak $b \neq 0, b(c-1)>0$ durumunda ise $O(0,0,0), \quad A_{1}(c-1, \sqrt{b(c-1)}, \sqrt{b(c-1)})$ ve $A_{2}(c-1,-\sqrt{b(c-1)},-\sqrt{b(c-1)})$ şeklinde üç denge noktasina sahip olur.

Teorem 3.1. $b>0$ için, (1) denklem sisteminin $O(0,0,0)$ denge noktası global üstel kararlıdır $\Leftrightarrow c<1$.

İspat: $X=(x, y, z)$ olsun.

$(\Leftarrow) \quad 0 \leq c<1$ için aşağıdaki pozitif tanımlı Lyapunov fonksiyonunu gözönüne alalım:

$$
W=\frac{1}{2}\left(x^{2}+\frac{1}{a} y^{2}+z^{2}\right)=\left[\begin{array}{c}
x \\
y \\
z
\end{array}\right]^{T}\left[\begin{array}{ccc}
1 / 2 & 0 & 0 \\
0 & 1 / 2 a & 0 \\
0 & 0 & 1 / 2
\end{array}\right]\left[\begin{array}{l}
x \\
y \\
z
\end{array}\right]=\left[\begin{array}{c}
x \\
y \\
z
\end{array}\right]^{T} P\left[\begin{array}{c}
x \\
y \\
z
\end{array}\right]
$$

Burada açıkça

$$
\min \left[\frac{1}{2 a}, \frac{1}{2}\right]\left(x^{2}+y^{2}+z^{2}\right) \leq W \leq \max \left[\frac{1}{2 a}, \frac{1}{2}\right]\left(x^{2}+y^{2}+z^{2}\right)
$$

yazılabilir. (1) sistemi boyunca $W$ nin türevi alınırsa,

$$
\begin{aligned}
\left.\frac{d W}{d t}\right|_{(1)} & =-b x^{2}-y^{2}-z^{2}+(c+1) y z \\
& =\left[\begin{array}{c}
x \\
y \\
z
\end{array}\right]^{T}\left[\begin{array}{ccc}
-b & 0 & 0 \\
0 & -1 & (c+1) / 2 \\
0 & (c+1) / 2 & -1
\end{array}\right]\left[\begin{array}{l}
x \\
y \\
z
\end{array}\right]=\left[\begin{array}{l}
x \\
y \\
z
\end{array}\right]^{T} Q\left[\begin{array}{l}
x \\
y \\
z
\end{array}\right] \\
& \leq \lambda_{\max }(Q)\left(x^{2}+y^{2}+z^{2}\right) \\
& \leq \max [(-1+c) / 2,(-3-c) / 2,-b]\left(x^{2}+y^{2}+z^{2}\right. \\
& =\max [(-1+c) / 2,-b]\left(x^{2}+y^{2}+z^{2}\right) \\
& \leq \frac{\max [(-1+c) / 2,-b]}{\max [1 / 2 a, 1 / 2]} W(X(t))
\end{aligned}
$$

olur. Buradan,

$$
W(X(t)) \leq W\left(X\left(t_{0}\right)\right) e^{\frac{\max [(-1+c) / 2,-b]}{\max [1 / 2 a, 1 / 2]}\left(t-t_{0}\right)}
$$

yazılabilir. Öte yandan, 


$$
\begin{gathered}
\min [1 / 2 a, 1 / 2]\left(x^{2}+y^{2}+z^{2}\right) \leq W(X(t)) \leq W\left(X\left(t_{0}\right)\right) e^{\frac{\max [(-1+c) / 2,-b]}{\max [1 / 2 a, 1 / 2]}\left(t-t_{0}\right)} \\
\quad \leq \max [1 / 2 a, 1 / 2]\left(x^{2}\left(t_{0}\right)+y^{2}\left(t_{0}\right)+z^{2}\left(t_{0}\right)\right) e^{\frac{\max [(-1+c) / 2,-b]}{\max [1 / 2 a, 1 / 2]}\left(t-t_{0}\right)}
\end{gathered}
$$

olur. Böylece,

$$
x^{2}(t)+y^{2}(t)+z^{2}(t) \leq \frac{\max [1 / 2 a, 1 / 2]}{\min [1 / 2 a, 1 / 2]}\left(x^{2}\left(t_{0}\right)+y^{2}\left(t_{0}\right)+z^{2}\left(t_{0}\right)\right) e^{\frac{\max [(-1+c) / 2,-b]}{\max [1 / 2 a, 1 / 2]}\left(t-t_{0}\right)} .
$$

elde edilir.

$c<0$ için, aşağıdaki gibi pozitif tanımlı, yeni bir Lyapunov fonksiyonunu gözönüne alalım:

$$
\tilde{W}=\frac{1}{2}\left(x^{2}-\frac{c}{a} y^{2}+z^{2}\right)
$$

Burada,

$$
\min [-c / 2 a, 1 / 2]\left(x^{2}+y^{2}+z^{2}\right) \leq \tilde{W} \leq \max [-c / 2 a, 1 / 2]\left(x^{2}+y^{2}+z^{2}\right)
$$

yazılabilir. (1) sistemi boyunca $\tilde{W}$ nin türevi alınırsa,

$$
\begin{aligned}
\left.\frac{d \tilde{W}}{d t}\right|_{(1)} & =-b x^{2}+c y^{2}-z^{2}=\left[\begin{array}{c}
x \\
y \\
z
\end{array}\right]^{T}\left[\begin{array}{ccc}
-b & 0 & 0 \\
0 & c & 0 \\
0 & 0 & -1
\end{array}\right]\left[\begin{array}{l}
x \\
y \\
z
\end{array}\right]=\left[\begin{array}{l}
x \\
y \\
z
\end{array}\right]^{T} R\left[\begin{array}{l}
x \\
y \\
z
\end{array}\right] \\
& \leq \lambda_{\max }(R)\left(x^{2}+y^{2}+z^{2}\right) \\
& \leq \max [-1,-b, c]\left(x^{2}+y^{2}+z^{2}\right. \\
& \leq \frac{\max [-1,-b, c]}{\max [-c / 2 a, 1 / 2]} \tilde{W}(X(t))
\end{aligned}
$$

elde edilir. Buradan

yazılabilir. Bu durumda

$$
\tilde{W}(X(t)) \leq \tilde{W}\left(X\left(t_{0}\right)\right) e^{\frac{\max [-1,-b, c]}{\max [-c / 2 a, 1 / 2]}\left(t-t_{0}\right)}
$$

$$
x^{2}(t)+y^{2}(t)+z^{2}(t) \leq \frac{\max [-c / 2 a, 1 / 2]}{\min [-c / 2 a, 1 / 2]}\left(x^{2}\left(t_{0}\right)+y^{2}\left(t_{0}\right)+z^{2}\left(t_{0}\right)\right) e^{\frac{\max [-1,-b, c]}{\max [-c / 2 a, 1 / 2]}\left(t-t_{0}\right)}
$$

elde edilir. Böylece (1) denklem sisteminin $O(0,0,0)$ denge noktası, global üstel kararlıdır.

$(\Rightarrow)(1.1)$ denklem sisteminin $O(0,0,0)$ denge noktası, global üstel kararlı olduğu için aynı zamanda yerel (locally) üstel kararlıdır. $O(0,0,0)$ noktasına karşılık gelen lineer hale dönüştürülmüş sistemin katsayı matrisi aşağıdaki gibi

$$
A=\left[\begin{array}{ccc}
-b & 0 & 0 \\
0 & -a & a \\
0 & c & -1
\end{array}\right]
$$


Hurwitz matrisidir $\Leftrightarrow A_{2 \times 2}=\left[\begin{array}{cc}-a & a \\ c & -1\end{array}\right]$ matrisi, $b>0$ olduğundan Hurwitz matrisidir. Burada $a-a c>0$ (yani $c<1$ ) olması Hurwitz matrisinin gerek ve yeter koşuludur. Bu ise ispatı tamamlar.

Teorem 3.2. (1) denklem sisteminin $O(0,0,0)$ denge noktası:

I. Eğer $c<1$ iken $b>0$ ise, asimptotik kararlıdır,

II. Eğer $b<0$ ise, kararsızdır,

III. Eğer $c<1$ iken herhangi bir $b$ değeri için karasızdır.

İspat: $O(0,0,0)$ noktasında (1) denklem sisteminin Jakobiyen (Jacobian) matrisi

$$
A=\left[\begin{array}{ccc}
-b & 0 & 0 \\
0 & -a & a \\
0 & c & -1
\end{array}\right]
$$

biçimindedir. Bu matrisin karakteristik polinomu,

$$
(\lambda+b)\left(\lambda^{2}+(a+1) \lambda+a-a c=0 .\right.
$$

olarak yazılır. $A$ matrisinin özdeğerleri, $\lambda_{1}=-b$ ve $\lambda_{2,3}=\frac{-(a+1) \pm \sqrt{(a+1)^{2}+4 a(c-1)}}{2}$ dir.

$A$ matrisinin özdeğerleri dikkate alınarak:

a) Ĕger $c<1$ iken $b>0$ ise, (1) denklem sisteminin $O(0,0,0)$ denge noktas1 global üstel kararlıdır (Teorem 3.1). Bu şartlar altında $A$ matrisinin özdeğerlerinin negatif olduğu sonucuna kolaylıkla ulaşılabilir. Böylece yukarıdaki $A$ matrisinin özdeğerlerinin tümü negatif reel kısımlı olduğundan (1) denklem sisteminin $O(0,0,0)$ denge noktası asimptotik kararlıdır.

b) Eğer $b<0$ ise, $A$ jakobiyen matrisi en az bir pozitif özdeğere sahiptir öyleki $\lambda_{1}=-b>0$ dır. O halde, (1) denklem sisteminin $O(0,0,0)$ denge noktası kararsızdır.

c) Eğer $c<1$ iken herhangi bir $b$ değeri için, $A$ jakobiyen matrisinin en az bir pozitif özdeğeri vardır. O halde, (1) denklem sisteminin $O(0,0,0)$ denge noktası kararsızdır.

Teorem 3.3. (1) denklem sisteminin

$$
A_{1}(c-1, \sqrt{b(c-1)}, \sqrt{b(c-1)}) \text { ve } A_{2}(c-1,-\sqrt{b(c-1)},-\sqrt{b(c-1)})
$$

denge noktaları asimptotik kararlıdır $\Leftrightarrow$

$$
a+b+1>0, \quad 2 a b(c-1)>0, \quad b(a+c)>0 \text { ve } b[(a+b+1)(a+c)-2 a(c-1)]>0
$$

dir.

İspat: $A_{1}(c-1, \sqrt{b(c-1)}, \sqrt{b(c-1)})$ noktasında (1) denklem sisteminin kararlı1ı̆̆ dikkate alındığında, (1) sistemi $(x, y, z) \rightarrow(x,-y,-z)$ dönüşümü altında değişmezdir (invariant’tır).

$(x, y, z) \rightarrow(X, Y, Z)$ lineer dönüşümü altında

olur. Buradan

$$
\left\{\begin{array}{l}
x=X+c-1 \\
y=Y+\sqrt{b(c-1)} \\
z=Z+\sqrt{b(c-1)}
\end{array}\right.
$$




$$
\left\{\begin{array}{l}
\dot{X}=\sqrt{b(c-1)}(Y+Z)-b X+Y Z \\
\dot{Y}=a(Z-Y) \\
\dot{Z}=-X \sqrt{b(c-1)}+Y-Z-X Y
\end{array}\right.
$$

yazılabilir. Bu durumda (6) denklem sisteminin kararlılığı dikkate alındığında, $O(0,0,0)$ noktasındaki jakobiyen matrisi

$$
J_{A_{1}}=\left[\begin{array}{ccc}
-b & \sqrt{b(c-1)} & \sqrt{b(c-1)} \\
0 & -a & a \\
-\sqrt{b(c-1)} & 1 & -1
\end{array}\right]
$$

olur. Bu matrisin karakteristik denklemi,

$$
\lambda^{3}+(a+b+1) \lambda^{2}+b(a+c) \lambda+2 a b(c-1)=0
$$

olarak yazılır. Routh-Hurwitz şartları dikkate alınarak, bu denklemin bütün köklerinin negative reel kısımlı olması için gerek ve yeter şart $P>0, Q>0, R>0$ ve $P Q-R>0$ olmasıdır. Burada, $Q=b(a+c), P=a+b+1$ ve $R=2 a b(c-1)$ dir.

Yani,

$$
\left\{\begin{array}{l}
a+b+1>0, \\
b(a+c)>0, \\
2 a b(c-1)>0 \\
b[(a+b+1)(a+c)-2 a(c-1)]>0
\end{array}\right.
$$

olarak yazılır. Böylece ispat tamamlanmış olur.

Uyarı 3.1 (1) denklem sistemindeki $a>0, b, c \in(-\infty,+\infty)$ şartları altında aşağıdaki gibi yeni bir $\dot{x}=y z-b x$,

$\dot{y}=a(z-y)$,

$\dot{z}=c y-x y$

denklem sistemi yazılabilir. Burada (7) sistemi, $a=15, b=8 / 3$ ve $c=30$ değerleri için kaotiktir (Şekil 2).

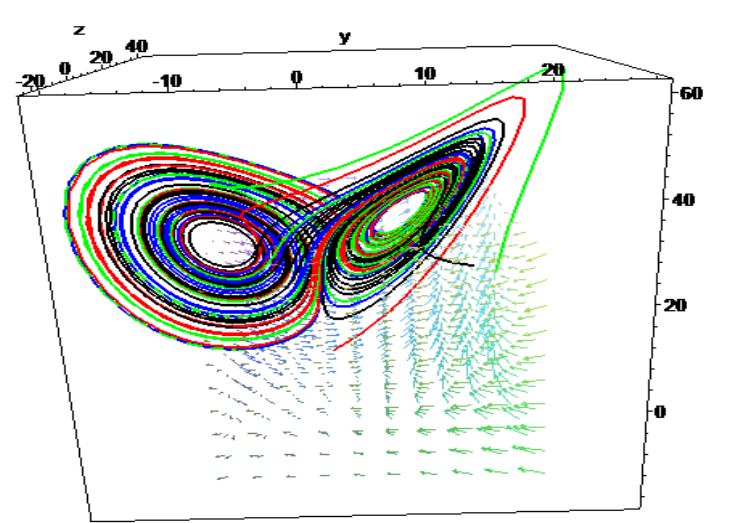

(c)

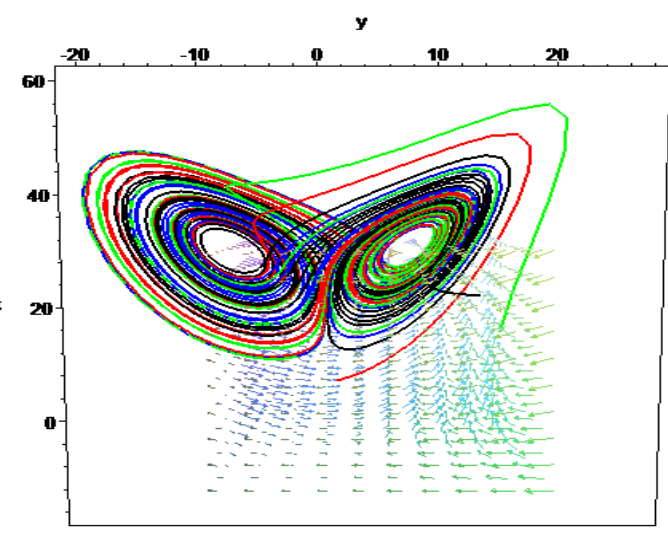

(d)

Şekil 2. (7) sisteminin üç boyutlu xyz-izdüşümü(c) ve xy-izdüşümü (d).

Önerme 3.2 (7) sistemini gözönüne alalım. Eğer $b \neq 0, b c>0$ ise, (7) denklem sistemi $O_{1}(0,0,0)$, $B_{1}(c, \sqrt{b c}, \sqrt{b c})$ ve $B_{2}(c,-\sqrt{b c},-\sqrt{b c})$ gibi üç ayrık denge noktasına sahip olur. Eğer $b \neq 0, b c \leq 0$ ise, (7) denklem sistemi yalnız bir $O_{1}(0,0,0)$ ayrık denge noktasına sahip olur. 
İspat: Bu önermenin ispat1, Önerme 3.1. in ispatına benzerdir.

Teorem 3.4 Her $c>0$ için, , (7) denklem sisteminin $O_{1}(0,0,0)$ denge noktası kararsızdır.

İspat: $O_{1}(0,0,0)$ noktasında $(7)$ denklem sisteminin jakobiyen matrisi

şeklindedir. Bu matrisin karakteristik denklemi,

$$
B=\left[\begin{array}{ccc}
-b & 0 & 0 \\
0 & -a & a \\
0 & c & 0
\end{array}\right]
$$

$$
(\lambda+b)\left(\lambda^{2}+a \lambda-a c\right)=0
$$

olarak yazılır. $B$ matrisinin özdeğerleri $\lambda_{1}=-b$ ve $\lambda_{2,3}=\frac{-a \pm \sqrt{a^{2}+4 a c}}{2}$ dir. Burada açıkça görülmektedir ki $c>0$ için $\lambda_{3}>0$ olur. Sonuç olarak $c>0$ için, (7) denklem sisteminin $O_{1}(0,0,0)$ denge noktası, karasızdır. Böylece, ispat tamamlanmış olur.

Teorem 3.5 Her $c \leq-1$ ve $b>0$ için, (7) denklem sisteminin $O_{1}(0,0,0)$ denge noktası, asimptotik kararlıdır.

İspat: $X=(x, y, z)$ olsun. Bu durumda aşağıdaki pozitif tanımlı Lyapunov fonksiyonunu gözönüne alalım:

$$
W=\frac{1}{2}\left(x^{2}+\frac{1}{a} y^{2}+z^{2}\right)
$$

(7) sistemi boyunca $W$ nin türevi alınırsa,

$$
\left.\frac{d W}{d t}\right|_{(2.3)}=-b x^{2}-y^{2}+(c+1) y z
$$

elde edilir. Her $c \leq-1$ ve $b>0$ için,

$$
\left.\frac{d W}{d t}\right|_{(2.3)} \leq 0
$$

olur. Bu takdirde (7) denklem sisteminin $O_{1}(0,0,0)$ denge noktası, asimptotik kararlı olur. Böylece ispat tamamlanmış olur.

Teorem 3.6 (7) denklem sisteminin $B_{1}(c, \sqrt{b c}, \sqrt{b c})$ ve $B_{2}(c,-\sqrt{b c},-\sqrt{b c})$ denge noktalarının asimptotik kararlı olması için gerek ve yeter şart $a+b>0, b(a+c)>0,2 a b c>0$ ve $b[(a+b)(a+c)-2 a c]>0$ olmasidır.

İspat: $B_{1}(c, \sqrt{b c}, \sqrt{b c})$ noktasında (7) denklem sisteminin kararlılığ dikkate alındığında, (7) sistemi $(x, y, z) \rightarrow(x,-y,-z)$ dönüşümü altında değişmezdir.

$(x, y, z) \rightarrow(X, Y, Z)$ lineer dönüşümü altında,

olur. Buradan

$$
\left\{\begin{array}{l}
x=X+c \\
y=Y+\sqrt{b c} \\
z=Z+\sqrt{b c}
\end{array}\right.
$$




$$
\left\{\begin{array}{l}
\dot{X}=\sqrt{b c}(Y+Z)-b X+Y Z \\
\dot{Y}=a(Z-Y) \\
\dot{Z}=-\sqrt{b c} X-X Y
\end{array}\right.
$$

yazılabilir. Bu durumda (8) denklem sisteminin kararlılığı dikkate alındığında, $O_{1}(0,0,0)$ noktasındaki jakobiyen matrisi

$$
J_{B_{1}}=\left[\begin{array}{ccc}
-b & \sqrt{b c} & \sqrt{b c} \\
0 & -a & a \\
-\sqrt{b c} & 0 & 0
\end{array}\right]
$$

olur. Bu matrisin karakteristik denklemi,

$$
\lambda^{3}+(a+b) \lambda^{2}+b(a+c) \lambda+2 a b c=0
$$

olarak yazılır. Routh-Hurwitz şartları dikkate alınarak, bu denklemin bütün köklerinin negatif reel kısımlı olması için gerek ve yeter şart $P>0, Q>0$ ve $P R-Q>0$ olmasıdır. Burada $P=a+b, Q=a b+b c$ ve $R=2 a b c$ dir.

Yani,

$$
\left\{\begin{array}{l}
a+b>0, \\
b(a+c)>0, \\
2 a b c>0 \\
b[(a+b)(a+c)-2 a c]>0
\end{array}\right.
$$

olarak yazilır. Böylece, ispat tamamlanmış olur.

\section{Sonuç}

Bu çalışmada, ilgili literatürde yapılan çalışmalar dikkate alınarak Lorenz benzeri üç boyutlu doğrusal olmayan yeni bir diferansiyel denklem sistemi analiz edildi. Çalışmada ilk olarak gözönüne alınan denklem sisteminin bazı katsayı değerleri için kaotik oldukları gösterildi. Daha sonra ele alınan sistemin denge noktaları belirlendi ve kararlılık kriterleri Routh-Hurwitz koşulları dikkate alınarak incelendi. Yine ele alınan sistemin üstel kararlılığı için Lyapunov fonksiyonundan yararlanılarak gerek ve yeter koşullar oluşturuldu. Sonuç olarak, elde edilen sonuçların ilgili literatüre katkı sağladığı ve bu doğrultuda çalışmaların genişletilebileceği düşünülmektedir.

\section{Kaynaklar}

[1] Al-Azzawi S.F. 2012. Stability and bifurcation of pan chaotic system by using Routh-Hurwitz and Gardan methods. Appl Math. Comput., 219: 1144-1152.

[2] Burton T.A. 1985. Stability and Periodic Solutions of Ordinary and Functional Differential Equations. Academic Press, Orlando, 337.

[3] Ertl J.P., Schafer E.W.P. 1969. Brain response Correlates of psychometric intelligence. Nature, 223: 421-422.

[4] Ha N.T.T., Strodiot J.J, Vuong P.T. 2018. On the global exponential stability of a projected dynamical system for strongly pseudomonotone variational inequalities. Optim Lett., 12: 16251638.

[5] Li D., Lu, J., Wu, X. 2005. Chen, G., Estimating the Bounds for the Lorenz family of chaotic systems. Chaos Solitons Fractals, 23 (2): 529-534.

[6] Li, D., Lu J., Wu X., Chen G. 2006. Estimating the ultimate bound and positively invariant set for the Lorenz System and a unified chaotic system, J. Math. Anal. Appl., 323 (2): 844-853.

[7] Li, D., Wu, X., Lu, J., 2009. Estimating the ultimate bound and positively invariant set for the hyperchaotic Lorenz-Haken system. Chaos Solitons Fractals, 39 (3): 1290-1296. 
[8] Li T., Chen G., Tang Y. 2004. On stability and bifurcation of Chen's system. Chaos, Solitons and fractals, 19 (5): 1269-1282.

[9] Lorenz, E.N. 1963. Deterministic non-periodic flow. J. Atmos. Sci., 20: 130-141.

[10] Lorenz E.N. 1963. The Essence of Chaos. Washington unv.

[11] Luo Q., Liao X.X., Zeng Z.G. 2010. Sufficient and Necessary Conditions for Lyapunov Stability of Lorenz System and their Application. Sci. China Inf. Sci., 53 (8): 1574-1583.

[12] Lü J., Chen G. 2002. A new chaotic attractor coined. International Journal of Bifurcation and Chaos, 12: 659-661.

[13] Pamuk N. 2013. Dinamik Sistemlerde Kaotik Zaman Dizilerinin Tespiti. BAÜ Fen Bil. Enst. Dergisi, 15 (1): 77-91.

[14] Ross S.L. 1984. Introduction to ordinary differential equations. Fourth edition, With the assistance of Shepley L. Ross II. John Wiley \& Sons, Inc., New York.

[15] Rizgar H.S. 2011. The Stability Analysis of the Shimizu-Morioka System with Hopf Bifurcation. Journal of Kirkuk University-Scientific Studies, 6 (2): 184-200.

[16] Rössler O.E. 1976. An Equation for Continuous Chaos. Phys . Lett. A 57: 397-398.

[17] Tigan G. 2005. Bifurcation and stability in a system derived from the Lorenz system. Balkan Society of Geometers, Geometry Balkan Press, 265-272.

[18] Ueta T., Chen G. 2000. Bifurcation analysis of Chen's equation. Internat. J. Bifur. Chaos Appl. Sci. Engrg., 10 (8): 1917-1931.

[19] Weiss H., Weiss V. 2003. The golden mean as clock cycle of brain waves. Chaos, Solitons and fractals, 18 (4): 1917-1931.

[20] Zhang F., Mu C., Li X. 2012. On the boundness of some solutions of the Lü system, Internat. J. Bifur. Chaos Appl. Sci. Engrg., 22 (1), 5 pp.

[21] Zhang F., Mu C., Zhou S., Zheng P. 2015. New results of the ultimate bound on the trajectories of the family of the Lorenz systems. Discrete Contin. Dyn. Syst. Ser. B 20 (4): 1261-1276.

[22] Zhang F., Shu Y., Yang H. 2011. Bounds for a new chaotic system and its application in chaos synchronization. Commun. Nonlinear Sci. Numer. Simul., 16 (3): 1501-1508. 